\title{
Incentives to Innovate in Oligopolies
}

P. Belleflamme and C. Vergari

Discussion Paper 2006-8

Département des Sciences Économiques

de l'Université catholique de Louvain 
CORE DISCUSSION PAPER 2006/14

\title{
Incentives to innovate in oligopolies*
}

\author{
Paul Belleflamme ${ }^{\dagger} \quad$ Cecilia Vergari $^{\ddagger}$
}

February 22, 2006

\begin{abstract}
In the spirit of Arrow (1962), we examine, in an oligopoly model with horizontally differentiated products, how much a firm is willing to pay for a process innovation that it would be the only one to use. We show that different measures of competition (number of firms, degree of product differentiation, Cournot vs Bertrand) affect incentives to innovate in non-monotonic, different, and potentially opposite ways.
\end{abstract}

Keywords: innovation, profit incentive, oligopoly, product differentiation.

JEL Classification Numbers: L13, 031.

${ }^{*}$ We are grateful to Jean Gabsziewicz, Antonio Minniti, Pierre Picard, Mario Tirelli and Vincent Vannetelbosch for useful comments and discussion about an earlier draft.

${ }^{\dagger}$ Corresponding author: CORE and Louvain School of Management, Université catholique de Louvain, 34 Voie du Roman Pays, B-1348 Louvain la Neuve, Belgium (tel: +32 104782 91, fax: +32 104743 01, e-mail: belleflamme@core.ucl.ac.be).

${ }^{\ddagger}$ CORE, Université catholique de Louvain, 34 Voie du Roman Pays, B-1348 Louvain la Neuve, Belgium and Department of Economics, University of Roma Tre, Rome, Italy (tel: +32 104743 58, fax: +32 104743 01, e-mail: vergari@core.ucl.ac.be). 


\section{Introduction}

Our objective in this paper is to explore in a systematic way how competition affects firms' incentives to innovate. Thereby, we aim at exploring further the relationship between market structure and innovation incentives, which has always been a central, and debated, issue in economics since Schumpeter's classic work, Capitalism, Socialism, and Democracy, in 1943.

Context. Schumpeter's first conjecture was to stress the necessity of tolerating the creation of monopolies as a way to encourage the innovation process. This argument is nothing but the economic rationale behind the legal protection of intellectual property and is nowadays widely accepted. Schumpeter's second conjecture was that large firms are better equipped to undertake R\&D than smaller ones. The best way to support this conjecture is probably to say that large firms have a larger capacity to undertake $\mathrm{R} \& \mathrm{D}$, insofar as they can deal more efficiently with the three market failures observed in innovative markets, namely externalities, indivisibilities and uncertainty. ${ }^{1}$ It is not clear, however, whether large firms, because of their monopoly power, also have larger incentives to undertake R\&D.

The pioneering paper studying the effect of market structure on the incentives for R\&D is Arrow (1962). He compares the profit incentive to innovate for monopolistic and competitive markets, concluding that perfect competition fosters more innovation than monopoly. The intuition behind this result is that a monopolist has less incentive to innovate because it already makes profit before the innovation, whereas the competitive firm just recoups its costs. This is the so called replacement effect: for the monopoly, the innovation just "replaces" an existing profit by a larger one. In Arrow's words, "the preinvention monopoly power acts as a strong disincentive to further innovation". As the following quote illustrates it, ${ }^{2}$ Arrow's intuition has made its way through the economic press:

\footnotetext{
${ }^{1}$ As far as externalities are concerned, large firms are likely to have less competitors able to imitate their innovation. In terms of indivisibilities, large firms are more qualified to exploit increasing returns in R\&D. Finally, regarding uncertainty, large firms are more diversified and, hence, more willing to take risks.

${ }^{2}$ Taken from "The meaning of XBox," The Economist, November 24, 2005.
} 
"It is surely no coincidence that Microsoft's hidden ability to innovate has become apparent only in a market in which it is the underdog and faces fierce competition. Microsoft is far less innovative in its core businesses, in which it has a monopoly (in Windows) and a near monopoly (in Office). But in the new markets of gaming, mobile devices and television set-top boxes, Microsoft has been unable to exploit its Windows monopoly other than indirectly - it has financed the company's expensive forays into pasture new."

Our approach. The previous argument seems to suggest that perfect competition also dominates, in terms of innovation incentives, oligopolistic market structures in which firms have some market power. However, this conjecture turns out to be wrong. We argue indeed that an intermediate form of competition may provide a higher incentive to innovate than the traditional polar cases (either monopoly or perfect competition). More generally, we examine how the intensity of competition affects incentives to innovate.

To this end, we consider an oligopoly model with horizontally differentiated products. In this setting, we address the same question as Arrow: how much is a firm willing to pay for a process innovation that it would be the only one to use? We also examine under which industry structure this willingness to pay reaches a maximum. To measure this willingness to pay, we compute the difference between the profit the firm would get by acquiring the innovation (and so reducing its marginal cost) and the profit the firm would get without the innovation. That is, we suppose that if the firm does not acquire the innovation, no other firm does. We measure thus the pure "profit incentive" to innovate, that is the desire to increase profits independently of the rival firms. As for the intensity of competition, our model allows us to consider different sources: the number of firms in the market, the degree of product differentiation and the nature of competition (Cournot vs Bertrand).

Results. The main finding of this analysis is that different industries are affected in qualitatively different ways by an increase in competition. The practical consequence is that, depending on the characteristics of the industry of interest, the highest profit incentive can be reached by a competitive 
firm (Arrow's claim), by a monopoly (Schumpeter's claim) or by an intermediate form of competition. We provide a rule about how to enhance firms' incentive to innovate for any industry.

More precisely, our main results can be summarized as follows. First, regarding the effect of product differentiation, the profit incentive is U-shaped whatever the nature of competition, the innovation size and the number of firms. Second, the effect of the number of firms depends on the other parameters. Under Cournot competition, the profit incentive either decreases with the number of firms or has a U-inverted shape. Under Bertrand competition, the profit incentive either decreases or increases with the number of firms. In both cases, for the second option to occur, the innovation and the degree of product substitutability must be large enough. Third, in both natures of competition, there exist ranges of parameters for which the profit incentive is affected in opposite ways by different measures of competition (it decreases with the number of firms and increases with the degree of product substitutability). Fourth, Arrow's result is no longer valid when products are sufficiently differentiated and/or the innovation is not too large; monopoly is then the optimal market structure in terms of profit incentive to innovate, which supports Schumpeter's second conjecture.

Related literature. Our framework is directly linked to a vast IO literature which, in line with Arrow, assumes that there is only one innovator which cannot be imitated by competitors. ${ }^{3}$ Bester and Petrakis (1993) contrast the profit incentive in Cournot and Bertrand duopolies for different degrees of horizontal product differentation. Bonanno and Haworth (1998) address the same question as Bester and Petrakis (1993) but under a model of vertical product differentiation. Yi (1999) examines the effect of the number of firms on the profit incentive to innovate in Cournot oligopolies with a homogeneous product. Delbono and Denicolò (1990) compare and contrast static and dynamic efficiency in oligopolies producing a homogeneous product for different number of firms. As Bester and Petrakis (1993), they use the nature of competition to measure the intensity of competition. They

\footnotetext{
${ }^{3}$ This literature as well as our paper abstract from the questions related to patent race. Our creative environment is characterized by what Scotchmer (2004) calls "scarce ideas" to distinguish them from ideas which are common knowledge.
} 
measure the incentive to innovate by the profit incentive as well as by the "competitive threat", i.e., the difference between the profit a firm gets with the innovation and the profit it would get if a rival firm found the innovation. The competitive threat comes from the recognition that there might be rival firms in the market competing to get the innovation. Here, the incentive depends on two sources of change of the profit: the profit incentive and the loss the firm would face in case any rival firm got the cost reduction. Hence, the value of the innovation increases because firms want to pay more in order to protect their position. Boone (2001) also studies the competitive threat but in a framework of asymmetric firms where he follows an axiomatic approach by defining as measure of competition a parameter satisfying particular conditions. ${ }^{4}$

One important conclusion that can be drawn from this literature is that different dimensions of competition may affect firms' investment in R\&D in non-monotonic and potentially different ways. Our contribution to this literature is to provide a unified framework where different sources of competition interact and determine firms' incentives to innovate. We not only confirm the results gathered in the previous literature, but we also extend some of them and, more importantly, we provide new results about the interaction between different measures of competition.

Our analysis can be related to two other strands of the literature on innovation. First, the incentives to innovate can be computed by including the revenue the innovator could raise through licensing the innovation. Additional issues arise then about the form the license should take (royalty per unit of output, fixed fee, ...) and about the number of licenses to be granted. Moreover, in an oligopoly setting, the identity of the innovator also matters (does the innovator compete on the product market or not?). Kamien (1992) surveys this literature and Kamien and Tauman (2002) extend it.

Second, another important question is how much firms are willing to invest in cost-reducing $\mathrm{R} \& \mathrm{D}$ (and not just how much they are willing to pay for an innovation of a given size), especially when all firms have the

\footnotetext{
${ }^{4}$ As pointed out in our conclusion, the study of the competitive threat in our framework constitutes one of our proposals for future research. However preliminary results do not show any qualitative difference with the profit incentive approach.
} 
simultaneous opportunity to achieve competing innovations. It must then be recognized that $\mathrm{R} \& \mathrm{D}$ is like any form of investment in that it precedes the production stage. As a result, strategic considerations and the extent of knowledge spillovers play a central role in determining which market structure provides firms with the highest incentives to undertake R\&D. The idea that $R \& D$ generates incentives for firms to behave strategically has been first examined by Brander and Spencer (1983), assuming no R\&D spillovers between firms and Cournot competition on the product market. Spence (1984), Okuno-Fujiwara and Suzumura (1990), and Qiu (1997) extended this analysis by considering, respectively, positive spillovers, Bertrand competition, and differentiated products. In these models, as opposed to our approach, each firm can obtain some cost reduction by investing in R\&D. In this symmetric setting, Cournot competition (with strategic substitutes) always results in higher cost reductions than Bertrand competition (with strategic complements), whereas in our framework, Bertrand competition leads to larger $R \& D$ investments than Cournot competition when product are close substitutes (as shown by Bester and Petrakis (1993) for the duopoly case). Regarding R\&D cooperation, d'Aspremont and Jacquemin (1988) presented a seminal analysis, which was then extended by, e.g, Kamien et al. (1992), and generalized by Amir et al. (2003).

The remainder of the paper is structured as follows. In Section 2, we set up the model. In Sections 3 and 4, we examine in turn Cournot and Bertrand competition. In Section 5, we define the optimal market structure in terms of incentives to innovate. We conclude in Section 6.

\section{Model}

There are $n$ firms (indexed by $i=1 \ldots n$ ) competing in the market. Each firm $i$ incurs a constant marginal cost equal to $c_{i}$ and produces a differentiated product, $q_{i}$, sold at price $p_{i}$. The demand system is obtained from the optimization problem of a representative consumer. We assume a quadratic utility function which generates the linear inverse demand schedule $p_{i}=a-q_{i}-\gamma \sum_{j \neq i} q_{j}$ in the region of quantities where prices are positive. The parameter $\gamma$ is an inverse measure of product differentiation: the lower 
$\gamma$ the more products are differentiated; in the limit if $\gamma=1$ products are perfect substitutes, if $\gamma=0$ products are perfectly differentiated and as a result demands are independent. The demand schedule, for $\gamma \neq 1$, is then given by $q_{i}=\alpha-\beta p_{i}+\delta \sum_{j \neq i} p_{j}$, with

$$
\alpha=\frac{a}{1+\gamma(n-1)}, \beta=\frac{1+\gamma(n-2)}{(1-\gamma)(1+\gamma(n-1))}, \delta=\frac{\gamma}{(1-\gamma)(1+\gamma(n-1))}
$$

Under Cournot competition, the equilibrium quantity and profit for firm $i$ are easily found as:

$$
q_{i}^{C}=\frac{(2-\gamma) a-(2+\gamma(n-2)) c_{i}+\gamma \sum_{j \neq i} c_{j}}{(2-\gamma)(2+\gamma(n-1))}, \pi_{i}^{C}=\left(q_{i}^{C}\right)^{2}
$$

We can also compute the equilibrium price, quantity and profit under Bertrand competition:

$$
\begin{aligned}
p_{i}^{B} & =\frac{(2 \beta+\delta) \alpha+\beta(2 \beta-\delta(n-2)) c_{i}+\delta \beta \sum_{j \neq i} c_{j}}{(2 \beta+\delta)(2 \beta-\delta(n-1))} \\
q_{i}^{B} & =\beta\left(p_{i}^{B}-c_{i}\right) ; \quad \pi_{i}^{B}=\beta\left(p_{i}^{B}-c_{i}\right)^{2}=(1 / \beta)\left(q_{i}^{B}\right)^{2}
\end{aligned}
$$

Initially, all firms produce at $\operatorname{cost} c_{i}=c$. A new process innovation allows firms to reduce the constant marginal cost of production from $c$ to $c_{1}=c-x$ (with $\left.0<x<c\right)$. We assume that the innovation is nondrastic. A sufficient condition is that the monopoly price corresponding to $c_{1}$ is larger than the initial cost $c$; that is, $(a+c-x) / 2>c$. Equivalently, assuming without loss of generality that the difference $a-c$ is equal to unity, we assume:

Assumption 1. $x<a-c=1 .^{5}$

In line with Arrow's question, we want to find out how much a firm is willing to pay for acquiring the innovation and being its single user. ${ }^{6}$ As a

\footnotetext{
${ }^{5}$ As shown by Zanchettin (2004) in the duopoly case, this condition is sufficient but not necessary for values of $\gamma<1$. We also assume that $c \geq 1$, so that Assumption 1 guarantees that the innovator still has a positive marginal cost.

${ }^{6}$ We discuss in the conclusion the role of the identity of the innovator (either incumbent or outside research lab).
} 
consequence, we concentrate on the profit incentive that can be seen as the "pure" incentive to innovate. We denote by $\pi_{W}$ the profit accruing to the innovator (the 'winner'), and by $\pi$ the current (pre-innovation) profit. For future reference, we also define $\pi_{L}$ as the profit accruing to the rivals of the innovator (the 'losers'). Based on these definitions, we formally define our measure of firms' incentives to innovate as follows:

Definition 1 The Profit Incentive is defined as $P I=\pi_{W}-\pi$.

We derive below the exact value of the profit incentive under Cournot and Bertrand competition, respectively noted $P I_{C}(n, \gamma)$ and $P I_{B}(n, \gamma)$. The profit incentive is clearly increasing in the innovation size, $x$. It also depends on the number of firms in the market $(n)$ and on the degree of product differentiation $(\gamma)$ in ways we will now analyse. Finally, note that

$$
P I_{C}(1, \gamma)=P I_{C}(n, 0)=P I_{B}(1, \gamma)=P I_{B}(n, 0)=\frac{x(x+2)}{4},
$$

which corresponds to the profit incentive for a monopoly (either because there is a single firm, $n=1$, or because products are independent, $\gamma=0$ ).

We want to study how the profit incentive changes with the intensity of competition. We consider three measures of the strength of competition: the degree of product substitutability $(\gamma)$, the number of firms in the market $(n)$, and the nature of competition (Cournot vs Bertrand).

As far as $\gamma$ is concerned, we know that as $\gamma$ decreases, firms' market power increases because products become more independent of each other. In the limit, when $\gamma$ is zero, we are in the presence of local monopolies. On the contrary as $\gamma$ approaches 1, profit tends to zero under Bertrand competition because we turn to perfect competition and decreases to a positive constant under Cournot competition with symmetric firms. Therefore, we study the effect of a change in the degree of product differentiation on the incentive to innovate. ${ }^{7}$

\footnotetext{
${ }^{7} \mathrm{~A}$ change in $\gamma$ can also be seen as a form of product innovation. Lin and Saggi (2002) distinguish the effects of such product innovation from those of a process innovation (like the one we consider here and which is usually modeled as a reduction in the marginal cost of production).
} 
The number of firms in the market is a measure of competition as long as firms are symmetric: in fact, as pointed out by Boone (2001), with asymmetric firms, an increase in competition, may force inefficient firms out of the market. As a result, a more competitive product market may imply a lower number of firms. In other words, when firms differ in their marginal costs, a lower $n$ does not necessarily mean a milder competition; note however that, on the other hand, a higher $n$ always means a tougher competition. In our framework, where firms are ex-ante symmetric, it can be easily shown that the current, the losers' and the winner's profits are decreasing in $n$. We then take $n$ as another measure of the strength of competition.

Finally, as for the nature of competition, from Singh and Vives (1984), we know that going from a Cournot to a Bertrand market structure implies an increase in competition.

\section{Cournot Competition}

In this section, we study how the incentives to innovate change with respect to the intensity of competition in a Cournot framework. We consider in turn the degree of product substitutability and the number of firms; we then examine the extent to which the effects of the two measures of competition converge or diverge.

Using expression (1), we find:

$$
\begin{aligned}
q_{W}^{C} & =\frac{(2-\gamma)+(2+\gamma(n-2)) x}{(2-\gamma)(2+\gamma(n-1))}, \pi_{W}^{C}=\left(q_{W}^{C}\right)^{2} \\
q^{C} & =\frac{1}{2+\gamma(n-1)}, \pi^{C}=\left(q^{C}\right)^{2}, \\
q_{L}^{C} & =\frac{(2-\gamma)-\gamma x}{(2-\gamma)(2+\gamma(n-1))}, \pi_{L}^{C}=\left(q_{L}^{C}\right)^{2},
\end{aligned}
$$

where Assumption 1 is used to guarantee that all equilibrium quantities (especially $q_{L}^{C}$ ) are always positive. Applying Definition 1, we compute the value of the profit incentive, as

$$
P I_{C}(n, \gamma)=\pi_{W}^{C}-\pi^{C}=x \frac{(2+\gamma(n-2))(2(2-\gamma)+(2+\gamma(n-2)) x)}{(2-\gamma)^{2}(2+\gamma(n-1))^{2}} .
$$

In order to analyse how $P I_{C}(n, \gamma)$ depends on $\gamma$ and $n$, we can get some intuition by first studying its separate components, $\pi_{W}^{C}$ and $\pi^{C}$. We 
summarize our results in the following lemma, where $\widehat{x}(n) \equiv 1 /(n+2)$ and $\hat{\gamma}(n, x) \equiv 2(x+1-\sqrt{x(x+n)}) /(1-x(n-2))$. The proof of this lemma and all subsequent proofs are relegated to the appendix.

Lemma 2 (i) The winner's and current profits both decrease with the number of firms in the market. (ii) The current profit decreases with the degree of product substitutability. (iii). The winner's profit increases with the degree of product substitutability if this degree and the size of the innovation are large enough (i.e., if $x>\widehat{x}(n)$ and $\gamma>\hat{\gamma}(n, x)$ ); it decreases otherwise.

It is worth stressing the third result in Lemma 2. In general profits are decreasing in $\gamma$. Nevertheless, if the innovation is large enough, the innovator may gain from an increase of $\gamma$. In particular, $\pi_{W}^{C}$ is first decreasing in $\gamma$ (because as soon as $\gamma$ becomes positive the firm is no longer a monopolist and remember that the innovation is non-drastic which implies that the innovation does not allow the innovator to become a monopolist) and then increasing in $\gamma$ (as soon as products are sufficiently substitutable, the cost advantage of the innovator becomes more important because the innovation becomes a sort of substitute for the product differentiation). This means that our two measures of competition have contrasting effects on the winner's profit. Note that $\widehat{x}(n)$ and $\hat{\gamma}(n, x)$ decrease with $n$. Therefore,the higher the number of firms in the market, the larger the range of parameters where the winner's profit is increasing with $\gamma$.

\subsection{Effect of product substitutability}

As we show in the next proposition, $P I_{C}$ first decreases with $\gamma$ and then increases. Whether the largest incentive is reached for $\gamma=0$ or $\gamma=1$ depends on the other parameters $(n$ and $x)$.

Proposition 3 Under Cournot competition, (i) the profit incentive is $U$ shaped with respect to $\gamma$. (ii) The highest profit incentive is reached under independent products $(\gamma=0)$ if the innovation size is below some threshold (comprised beween $2 / 7$ and $2 / 3$ ), and under homogeneous products $(\gamma=1$ ) otherwise. 
The proposition is formally stated and proved in the appendix. While the proof is technical, we now give the intuition behind the U-shaped form. First, it is useful to consider an infinitesimal reduction in cost $(x \rightarrow 0)$ and use calculus. Indeed, the profit incentive can be seen as the discrete version of the sensitivity of $i$ 's profits to a reduction in its own $\operatorname{cost}\left(\right.$ i.e., $-\partial \pi_{i}^{C} / \partial c_{i}$ evaluated at $c_{i}=c$ ). Decomposing the latter derivative, we have:

$$
\begin{aligned}
-\frac{\partial \pi_{i}^{C}}{\partial c_{i}} & =2 q_{i}^{C}(\gamma) \cdot F(\gamma), \\
\text { with } F(\gamma) & \equiv\left(-\frac{\partial q_{i}^{C}}{\partial c_{i}}\right)=\frac{2+\gamma(n-2)}{(2-\gamma)(2+\gamma(n-1))}>0 .
\end{aligned}
$$

To explain the U-shape, we are interested in establishing the sign of

$$
-\frac{\partial^{2} \pi_{i}^{C}}{\partial c_{i} \partial \gamma}=2 \frac{\partial q_{i}^{C}}{\partial \gamma} F(\gamma)+2 \frac{\partial F}{\partial \gamma} q_{i}^{C}(\gamma)
$$

From Lemma 2, we know that $\partial q_{i}^{C} / \partial \gamma$ is negative. We can also easily show that, in contrast, $\partial F / \partial \gamma$ is positive for $\gamma>0$ and is equal to zero for $\gamma=0$. This means that as products become closer substitutes, there are two contrasting effects: the equilibrium quantity decreases but becomes more sensitive to a reduction in the firm's own marginal cost. In other words, comparing two situations, $\gamma_{1}>\gamma_{2}$, we have that a cost reduction produces a larger change in quantity under $\gamma_{1}$ than under $\gamma_{2}$, but this change also applies to a smaller quantity. The net effect is thus ambiguous. However, we have clear cut results for the extreme values of $\gamma$. When $\gamma=0$, the second effect disappears and so, the marginal return of a cost reduction decreases with $\gamma$. At the other extreme $(\gamma=1)$, it can be shown that the second effect dominates the first effect and so, the marginal return of a cost reduction increases with $\gamma$. There is thus an intermediate value of $\gamma$, for which the two effects just compensate and where $-\partial \pi_{i}^{C} / \partial c_{i}$ reaches a minimum.

Let us now extend the previous argument to the discrete case. We can rewrite the profit incentive as

$$
P I_{C}=\pi_{W}^{C}-\pi^{C}=\left(q_{W}^{C}\right)^{2}-\left(q^{C}\right)^{2}=\left(q_{W}^{C}+q^{C}\right)\left(q_{W}^{C}-q^{C}\right) .
$$

Comparing expressions (8) and (9), we can say that $2 q_{i}^{C}(\gamma)$ approximates $\left(q_{W}^{C}+q^{C}\right)$, and $F(\gamma)$ approximates $\left(q_{W}^{C}-q^{C}\right)$. Naturally, in the discrete 
case, the way these terms change with $\gamma$ depends on the value of the cost reduction. As far as $\left(q_{W}^{C}-q^{C}\right)$ is concerned, we can show that it behaves like $F(\gamma)$ in the sense that it increases with $\gamma$ whatever the innovation size. However, $\left(q_{W}^{C}+q^{C}\right)$ might behave differently than $2 q_{i}^{C}(\gamma)$ : if the cost reduction and the degree of product substitutability are large enough, $\left(q_{W}^{C}+q^{C}\right)$ increases with $\gamma$. (This is because of the effect of the winner's quantity, as explained in Lemma 2.) It thus means that for large values of $\gamma$ the two effects are positive, which just reinforces the conclusion we drew from the infinitesimal case.

The fact that larger values of $\gamma$ lead to a larger profit incentive to innovate can be seen as a form of substitutability between product innovation (modeled as a reduction of $\gamma$ ) and process innovation (measured by $x$ ): the weaker the product innovation, the higher the willingness to pay for a given process innovation.

\subsection{Effect of the number of firms}

The increase in the number of firms has two opposite effects on the incentive to innovate. On the one hand, there is a competition effect: a larger number of firms reduces firm $i$ 's profit if either it gets the innovation or it does not. On the other hand, there is a competitive advantage: the higher $n$, the higher the number of rival firms producing in a less efficient way. This in turn implies that, in some cases, the effect of the number of firms on the profit incentive is also non-monotonic.

Proposition 4 (i) The profit incentive is a single-peaked function of $n$. (ii) If the innovation size and the degree of product substitutability are large enough, then the maximum value of $P I$ is reached for $n>1$ (competition); otherwise, the maximum value of $P I$ is reached for $n=1$ (monopoly).

Again, we formally state and prove the proposition in the appendix. ${ }^{8}$ The interesting result is that when $x$ and $\gamma$ are large enough, the profit in-

\footnotetext{
${ }^{8}$ This result complements and extends Yi (1999) who focuses on homogeneous product markets. For an infinitesimal innovation, Yi shows that the profit incentive decreases with $n$ for a fairly large class of demand functions. He also considers arbitrary innovations under linear demand and finds the same threshold value for the innovation size as we do.
} 
centive to innovate first increases and then decreases with $n$ : for a sufficiently low number of firms, the competitive advantage dominates the competition effect; that is, an extra firm in the market implies a larger gain from getting the innovation because one more firm faces a higher marginal cost; clearly, this gain is larger the less differentiated are the products and the larger is the innovation size. In contrast, when the number of firms in the market reaches a certain threshold, the overall effect on the profit incentive becomes negative. We check the presence of these two effects by deriving expression (9) with respect to $n$ :

$$
\begin{aligned}
\frac{\partial}{\partial n} P I_{C}(\gamma, n)= & \underbrace{\frac{\partial\left(q_{W}^{C}+q^{C}\right)}{\partial n}}_{\text {competition effect (-) }}\left(q_{W}^{C}-q^{C}\right) \\
& +\underbrace{\frac{\partial\left(q_{W}^{C}-q^{C}\right)}{\partial n}}_{\text {competitive advantage }}\left(q_{W}^{C}+q^{C}\right) .
\end{aligned}
$$

Performing a similar infinitesimal analysis as above, we confirm that for small values of $x(x \rightarrow 0)$, the competition effect always dominates the competitive advantage, and the profit incentive decreases with $n$; indeed, we compute:

$$
-\frac{\partial^{2} \pi_{i}^{C}}{\partial c_{i} \partial n}<0
$$

At the other extreme, we can show that when $\gamma$ and $x$ tend to one (i.e., towards homogeneous product and drastic innovation), the profit incentive is always increasing with the number of firms.

\subsection{Cross-effects}

We want now to contrast the ways the two measures of competition affect the profit incentive. According to Proposition 4, the profit incentive increases with the number of firms provided that the innovation size and the degree of product substitutability are large enough. Let us make this statement more 
precise. Considering $n$ as a continuous variable, it is readily shown that

$$
\frac{\partial}{\partial n} P I_{C}(n, \gamma)>0 \Leftrightarrow \gamma>\bar{\gamma}(n, x)=\frac{n-x-4+\sqrt{(n+x)^{2}-4(n-1)}}{n-3+n x-2 x},
$$

where $\bar{\gamma}(n, x)>0$ for all admissible $n$ and $x$, and $\bar{\gamma}(n, x)<1$ if and only if $x>(n-1) / n$.

From Proposition 3, we know that the profit incentive is U-shaped with respect to $\gamma$. Let $\tilde{\gamma}(n, x)$ denote the value of $\gamma$ for which the profit incentive reaches its minimum (we proved that $0<\tilde{\gamma}(n, x)<1$ for all admissible $n$ and $x)$. We find that $\bar{\gamma}(n, x)>\tilde{\gamma}(n, x)$ for all admissible $n$ and $x$, which allows us to state the following proposition.

Proposition 5 (i) The two measures of competition affect the profit incentive in converging ways either if products are sufficiently differentiated (for $\gamma<\tilde{\gamma}(n, x)$, the profit incentive decreases with $\gamma$ and $n)$, or if the innovation is large enough and products are sufficiently substitutes (for $x>(n-1) / n$ and $\gamma>\bar{\gamma}(n, x)$, the profit incentive increases with $\gamma$ and $n)$. (ii) There always exists a range of parameter values for which the two measures of competition affect the profit incentive in diverging ways (for $\tilde{\gamma}(n, x)<\gamma<$ $\min \{\bar{\gamma}(n, x), 1\}$, the profit incentive increases with $\gamma$ and decreases with $n)$.

The results of Proposition 5 gives us a rule about how to enhance the incentives to innovate for any innovation size $x$ and any industry $(n, \gamma)$. For instance, it tells us for which industries, a merger of two firms (decrease in $n$ ) or the introduction of a product innovation (decrease in $\gamma$ ) increase or decrease the incentive to invest in a process innovation.

Another implication of Proposition 5 is that the same level of profit incentive can be achieved in different industries. In this respect, an instructive thought experiment is to fix the innovation size $x$ and examine which industries give firms the same level of profit incentive as a monopoly ( $n=1$ and/or $\gamma=0)$. We know from Proposition 3 that for $x<2 / 7$, the monopoly gives a higher profit incentive than any other industry. So, we take for example $x=1 / 2$ and we compute that the following industries $(n, \gamma)$ are equivalent to a monopoly in terms of profit incentive: $(2,0.89),(3,0.94),(4,0.98)$, and 
$(5,1) .{ }^{9}$ We observe that to maintain the profit incentive at the monopoly level, an increase in the number of firms has to be compensated by an increase in product substitutability. In particular, we observe that five firms producing a homogeneous product are willing to pay the same amount than a monopoly for a process innovation that decreases the marginal cost of production by $x=1 / 2$.

\section{Bertrand Competition}

The derivation of the profit incentive is slightly more involved under Bertrand competition. Indeed, in contrast with Cournot competition, Assumption 1 is not sufficient to ensure that the non-innovating firms (the 'losers') are all active on the market after the innovation. In fact, if firm $i$ is less efficient than firm $j$ (because of the innovation $x>0$ ), it sets a price larger than its marginal cost $c$ (and thus produces a positive quantity) as long as its product is sufficiently different from $j$ 's product. We need thus to consider the possibility of corner equilibria in which a number of losers are constrained to price at marginal cost. The following lemma characterises the BertrandNash equilibrium of the post-innovation game for all values of $x, n$, and $\gamma$. Define

$$
x_{B}(n, \gamma)=\frac{(1-\gamma)(2 \gamma n-3 \gamma+2)}{\gamma(1+\gamma n-2 \gamma)} .
$$

Lemma 6 The Nash equilibrium of the post-innovation price game is such that (i) for $x \leq x_{B}(n, \gamma)$, all firms price above their marginal cost, and (ii) for $x>x_{B}(n, \gamma)$, the winner is the only firm pricing above its marginal cost.

Note that the threshold separating the two cases, $x_{B}(n, \gamma)$, decreases with $n$ and tends, for $n \rightarrow \infty$, to $2(1-\gamma) / \gamma$, which is greater than unity for $\gamma<2 / 3$. Therefore, the corner solution can only be observed for sufficiently large values of $x$ and $\gamma$.

\footnotetext{
${ }^{9}$ The larger $x$, the more "equivalent" industries can be found. At the limit, when the innovation becomes drastic $(x=1)$, there is an infinity of "equivalent" industries.
} 
We can now define the profit incentive. First, from expressions (2) and (3), we derive the current profit:

$$
\pi^{B}(n, \gamma)=\frac{1+\gamma(n-2)}{(1-\gamma)(1+\gamma(n-1))}\left(\frac{1-\gamma}{\gamma(n-3)+2}\right)^{2} .
$$

Next, using the proof of Lemma 6 , we compute the winner's profit at the Nash equilibrium of the post-innovation game:

$$
\begin{cases}\pi_{W}^{B}(n, \gamma)=\beta\left(\frac{\left(\left(5-5 n+n^{2}\right) \gamma^{2}+3(n-2) \gamma+2\right) x+(1-\gamma)(2+2 \gamma n-3 \gamma)}{(2+\gamma n-3 \gamma)(2+2 \gamma n-3 \gamma)}\right)^{2} \\ \bar{\pi}_{W}^{B}(\gamma)=\left(1-\frac{1}{\gamma}+x\right) \frac{1}{\gamma} & \text { for } x \leq x_{B}(n, \gamma), \\ & \text { for } x>x_{B}(n, \gamma) .\end{cases}
$$

Finally, combining (11) and(12), we can express the profit incentive under Bertrand competition as

$$
P I_{B}(n, \gamma)= \begin{cases}\underline{P I}_{B}(n, \gamma)=\pi_{W}^{B}(n, \gamma)-\pi^{B}(n, \gamma) & \text { for } x \leq x_{B}(n, \gamma) \\ \overline{P I}_{B}(n, \gamma)=\bar{\pi}_{W}^{B}(\gamma)-\pi^{B}(n, \gamma) & \text { for } x>x_{B}(n, \gamma)\end{cases}
$$

We can now examine how the profit incentive evolves with the two measures of competition. Starting with product substitutability, we observe that its effect is non-monotonic, as described in the following proposition.

Proposition 7 Under Bertrand competition, (i) the profit incentive first decreases with $\gamma$, then reaches a minimum, and finally increases with $\gamma$. (ii) The highest profit incentive is reached under homogeneous products $(\gamma=1)$ for all $n$ and $x$.

We observe thus that the degree of product substitutability affects $P I_{B}$ and $P I_{C}$ in qualitatively similar ways. Bester and Petrakis (1993) compare $P I_{B}$ and $P I_{C}$ in a duopoly model by restricting the range of $\gamma$ to values such that all firms stay on the market (i.e., $x \leq x_{B}(2, \gamma)$ ). They show that there exists a cutoff value of $\gamma$ such that $P I_{C}>P I_{B}$ for $\gamma$ below the cutoff and $P I_{B}>P I_{C}$ for $\gamma$ above the cutoff. Using our derivation of $\overline{P I}_{B}(2, \gamma)$, we can show that Bester and Petrakis' conclusion extends to larger values of 
$\gamma$ (i.e., those for which an ex post monopoly obtains). ${ }^{10}$ Moreover, we show that under Bertrand competition, the profit incentive reaches its maximum for homogeneous products independently of the other parameters $(n$ and $x$ ).

On the other hand, the effect of the number of firms on the profit incentive under Bertrand competition is monotone. As we show in the next proposition, $P I_{B}$ is either decreasing or increasing with $n$ depending on the innovation size and the degree of product differentiation. We show in Appendix 7.7 that there is a unique positive value of $x, \bar{x}(n, \gamma)$, such that $\partial \underline{P I}_{B}(n, \gamma) / \partial n=0$, and that $\bar{x}(n, \gamma)<x_{B}(n, \gamma)$ for all $n$ and $\gamma$. Therefore, we can state:

Proposition 8 Under Bertrand competition, the profit incentive decreases with the number of firms for $x<\bar{x}(n, \gamma)$, and increases otherwise. A necessary condition for the latter possibility $(\bar{x}(n, \gamma)<1)$ is $\gamma>0.65$.

Let us now provide some intuition for these results. As for Cournot, we first proceed with the infinitesimal analysis. The profit incentive can be seen as the discrete version of the sensitivity of $i$ 's profits to a reduction in its own cost (i.e., $-\partial \pi_{i}^{B} / \partial c_{i}$ ). Decomposing the latter derivative, we have:

$$
\begin{aligned}
-\frac{\partial \pi_{i}^{B}}{\partial c_{i}} & =2 \frac{q_{i}^{B}}{\beta} \cdot G(\gamma, n) \\
\text { with } G(\gamma, n) & \equiv\left(-\frac{\partial}{\partial c_{i}} q_{i}\right)=\frac{(1+\gamma(n-2))\left(2+3 \gamma n-6 \gamma+\gamma^{2} n^{2}-5 \gamma^{2} n+5 \gamma^{2}\right)}{(2+\gamma n-3 \gamma)(2+2 \gamma n-3 \gamma)(1-\gamma)(1+\gamma n-\gamma)}>0 \\
\text { and } \frac{q_{i}^{B}}{\beta} & =\left(p_{i}^{B}-c_{i}\right)=\frac{1-\gamma}{2+\gamma n-3 \gamma} .
\end{aligned}
$$

To explain the effect of competition on $-\partial \pi_{i}^{B} / \partial c_{i}$, we are interested in establishing the sign of

$$
\begin{aligned}
\frac{\partial}{\partial \gamma}\left(-\frac{\partial}{\partial c_{i}} \pi_{i}^{B}\right) & =2 G \cdot \frac{\partial}{\partial \gamma}\left(p_{i}^{B}-c_{i}\right)+2\left(p_{i}^{B}-c_{i}\right) \cdot \frac{\partial}{\partial \gamma} G, \\
\frac{\partial}{\partial n}\left(-\frac{\partial}{\partial c_{i}} \pi_{i}^{B}\right) & =2 G \cdot \frac{\partial}{\partial n}\left(p_{i}^{B}-c_{i}\right)+2\left(p_{i}^{B}-c_{i}\right) \cdot \frac{\partial}{\partial n} G,
\end{aligned}
$$

where

$$
\begin{aligned}
\frac{\partial}{\partial \gamma}\left(p_{i}^{B}-c_{i}\right) & =-\frac{n-1}{(2+\gamma n-3 \gamma)^{2}}<0, \text { and } \frac{\partial}{\partial \gamma} G(\gamma, n) \geq 0, \\
\frac{\partial}{\partial n}\left(p_{i}^{B}-c_{i}\right) & =\frac{-(1-\gamma) \gamma}{(2+\gamma n-3 \gamma)^{2}}<0 \text { and } \frac{\partial}{\partial n} G(\gamma, n)>0 .
\end{aligned}
$$

\footnotetext{
${ }^{10}$ Simulations indicate that these results still hold for $n>2$.
} 
Therefore, as competition becomes fiercer, that is as $\gamma$ and $n$ increase, two opposite forces are involved: on the one hand competition has a negative effect on the price cost margin, on the other hand it has a positive effect on profits' sensitivity to cost. However, in contrast with Cournot competition, when $x \rightarrow 0$ the negative effect always overcompensates the positive one; in fact:

$$
\frac{\partial}{\partial \gamma}\left(-\frac{\partial}{\partial c_{i}} \pi_{i}^{B}\right)<0 \text { for any } \gamma \text {, and } \frac{\partial}{\partial n}\left(-\frac{\partial}{\partial c_{i}} \pi_{i}^{B}\right)<0 \text { for any } n .
$$

Let us now extend the previous argument to the discrete case. We can rewrite the profit incentive as

$$
P I_{B}=\pi_{W}^{B}-\pi^{B}=\frac{1}{\beta}\left(q_{W}^{B}+q^{B}\right)\left(q_{W}^{B}-q^{B}\right),
$$

where $\left(q_{W}+q\right) / \beta$ approximates $2 q^{B} / \beta$ and $\left(q_{W}-q\right)$ approximates $G(\gamma, n)$. We first study how these components change with $\gamma$. From Appendix 7.6, we can see that they behave like their corresponding infinitesimal terms.

On the other hand, the discrete analysis with respect to $n$, changes with the innovation size. In particular, we show that $\left(q_{W}^{B}-q^{B}\right)$ behaves like $G(\gamma, n)$, that is it increases with $n$ whatever the innovation size; however, $\left(q_{W}^{B}+q^{B}\right) / \beta$ might behave differently than $2 q_{i}^{B} / \beta$ : if the cost reduction and the degree of product substitutability are large enough, $\left(q_{W}^{B}+q^{B}\right)$ increases with $n$.

As far as the cross effects of the two measures of competition are concerned, the picture is similar to the one we described for Cournot competition. Defining $\underline{x}(n, \gamma)$ as the positive value of $x$ such that $\partial P I_{B}(n, \gamma) / \partial \gamma=$ 0 , we can show that $\underline{x}(n, \gamma)<\bar{x}(n, \gamma)$, meaning that there are up to three possible situations: (i) for $x<\underline{x}(n, \gamma)$, the profit incentive decreases with both $n$ and $\gamma$; (ii) for $\underline{x}(n, \gamma)<x<\bar{x}(n, \gamma)$, the profit incentive decreases with $n$ but increases with $\gamma$; (iii) for $x>\bar{x}(n, \gamma)$, the profit incentive increases with both $n$ and $\gamma$. As for Cournot competition, it is impossible to have the profit incentive increase with $n$ while decreasing with $\gamma$. 


\section{Optimal Market Structure}

After studying how different sources of competition affect the profit incentive to innovate, we can proceed to investigate what is the optimal market structure in terms of profit incentives to innovate. Notice that we are not looking for the welfare maximizing industry, nor for the "welfare incentive" to innovate. In contrast, starting from the idea that a process innovation is welfare enhancing (it implies an increase in total industry profits as well as an increase in consumer surplus), we compared how much a firm is willing to pay for a process innovation.under different market structures (perfect competition, oligopolies, monopoly). We now wonder under which conditions this willingness to pay is maximised.

Arrow (1962)'s argument about the optimal market structure in terms of profit incentive was based on Bertrand competition in a homogeneous market. We check indeed that with a homogeneous product, the profit incentive under Bertrand competition is defined as $\overline{P I}_{B}(n, 1)$, which increases in $n$ and reaches thus its maximum at $\overline{P I}_{B}(\infty, 1)=x$. It is easily checked that $\overline{P I}_{B}(\infty, 1)=x>P I_{B}(1,1)=(x+2) x / 4$, which establishes Arrow's result.

Now, Proposition 4 tells us that the previous argument does not hold under Cournot competition. Indeed, letting the number of firms going to infinity never leads to the highest profit incentive. Also, Proposition 8 shows that as soon as products are differentiated, the profit incentive under Bertrand competition might be decreasing in $n$ if the innovation is small enough, meaning that the monopoly gives higher incentives to innovate.

Combining our previous results, we are in a position to complement Arrow's analysis in a useful way by solving the following exercise: for a given degree of product differentiation and a given innovation size, what is the combination of competition mode and number of firms that yields the highest profit incentive to innovate? As shown in the following proposition, the answer is very simple to state.

Proposition 9 For sufficiently large values of $x$ and $\gamma$, Bertrand competition with an infinite number of firms yields the highest profit incentive. Otherwise, (Bertrand or Cournot) monopoly does. 
More formally (as proved in Appendix 7.8), for $x>2(1-\gamma) / \gamma$ (which supposes $\gamma>2 / 3$ ), the best structure under Bertrand competition (that is, the optimal number of firms $n_{B}^{*} \rightarrow \infty$ ) yields a higher profit incentive than the best structure under Cournot competition (i.e., $n_{C}^{*}=1$ or $n_{C}^{*}$ is (the integer closest to) $\hat{n}(x, \gamma)$ depending on the value of $x$ ). On the other hand, for $x<2(1-\gamma) / \gamma$, the optimal market structure is monopoly, which independently of the nature of competition provides a profit incentive given by $(x+2) x / 4$.

\section{Conclusion}

We have provided a unified framework where different sources of competition interact and determine firms' incentives to invest in a process innovation.

Our results are twofold: on the one hand, we confirm the existence of a non-monotone and non-unique relationship between the intensity of competition and the incentives to innovate in a general framework; in particular, we extend the analysis of the profit incentive to innovate under Bertrand competition with linear demand and horizontally differentiated products by considering any number of firms and any degree of product differentiation. On the other hand, we show that different sources of competition can have diverging effects on the innovation incentives; we characterize the conditions under which this occurs, thus providing a rule about how to enhance firms' incentives to innovate.

In the spirit of Arrow (1962), we focus our attention on the profit incentive as a measure of firms' innovation incentives. This strategy allows us to extend and complement Arrow's analysis; in contrast, we do not consider the incentive which comes from the recognition that there exist rival firms competing to be the first to innovate, that is the competitive threat. The analysis of the competitive threat in such a framework constitutes a first possible extension of our model. Nevertheless, preliminary results show that the different sources of competition considered here affect the competitive threat and the profit incentive in qualitatively similar ways.

A second linked research area we propose to work on deals with the identity of the innovator. Our profit incentive may correspond to either of the 
following two scenarios. We can think first that some outside research lab has discovered the innovation and auctions a single license to the highest bidder; our question was to evaluate what the highest bid would be. Alternatively, we can imagine that the innovator is an incumbent firm, which either manages to keep its innovation secret, or which is granted a broad patent of infinite duration and which does not license the innovation to rival firms; our question was then to assess how much this incumbent is willing to invest in R\&D. Nevertheless, our approach does not take into account the incentive of the innovator to license his cost-reducing innovation. In particular, Kamien and Tauman (2002) demonstrate that an outside innovator finds it profitable to auction more than one license, and that an inside innovator also has incentives to license the innovation to its rivals. Their approach is an alternative measure of firms' incentives to innovate, that is the innovator's profit coming from the mode of licensing and the number of licenses auctioned off. We leave it to future research to investigate whether their results still hold in the presence of product differentiation. Studying the behavior of the innovator in our framework would allow us to have a more complete picture of firms' innovation incentives in oligopolistic settings.

\section{Appendix}

\subsection{Proof of Lemma 2}

(i) It is obvious from (6) and (5) that both $\pi_{L}^{C}$ and $\pi^{C}$ decrease with the number of firms. As for the winner's profit, we consider the number of firms as a continuous variable. We compute the sign of the first-order derivative of the equilibrium profit with respect to $n$. It is readily seen that $\partial \pi_{W}^{C} / \partial n$ is negative if and only if $\gamma<2 /(x+1)$, which is clearly satisfied as $\gamma, x<1$.

(ii) It is obvious from (5) that $\pi^{C}$ decreases with $\gamma$. As for the loser's profit, we compute the first-order derivative with respect to $\gamma$ :

$$
\frac{\partial}{\partial \gamma} \pi_{L}^{C}=-\frac{\left(\gamma^{2}(n-1)+4\right) x+(2-\gamma)^{2}(n-1)}{(2-\gamma)^{2}(2+\gamma n-\gamma)^{2}}\left(2 q_{L}^{C}\right)<0 .
$$

(iii) We compute the first-order derivative of the winner's profit with 
respect to $\gamma$ :

$$
\frac{\partial}{\partial \gamma} \pi_{W}^{C}=\left(2 q_{W}^{C}\right) \frac{(n-1)\left(\gamma(\gamma(n-2)+4) x-(2-\gamma)^{2}\right)}{(2-\gamma)^{2}(2+\gamma n-\gamma)^{2}} .
$$

The derivative has the same sign has the following second-degree polynomial in $\gamma$ : $((n-2) x-1) \gamma^{2}+4(x+1) \gamma-4$. This polynomial has two real roots:

$$
\gamma_{1}=2 \frac{x+1+\sqrt{x(n+x)}}{1-x(n-2)}, \gamma_{2}=2 \frac{x+1-\sqrt{x(n+x)}}{1-x(n-2)} \text {. }
$$

We need to distinguish between two cases. First, if $x>1 /(n-2)$, it is easily checked that $\gamma_{1}<0<\gamma_{2}<1$. Therefore, the polynomial is positive for $\gamma \in\left[\gamma_{2}, 1\right]$. Second, for $x<1 /(n-2)$, two sub-cases must be considered. (A) If $1 /(n+2)<x<1 /(n-2)$, then one checks that $0<\gamma_{2}<1<\gamma_{1}$ and the polynomial is positive for $\gamma \in\left[\gamma_{2}, 1\right]$. (B) If $x<1 /(n+2)$, then one checks that $1<\gamma_{2}<\gamma_{1}$ and the polynomial is negative for all $\gamma \in[0,1]$.

We can summarize the previous results as follows. Let $\widehat{x} \equiv 1 /(n+2)$ and $\hat{\gamma} \equiv 2 \frac{x+1-\sqrt{x(x+n)}}{1-x(n-2)}$. For $x<\widehat{x}$, the winner's profit decreases with $\gamma$. For $x>\widehat{x}$, the winner's profit decreases with $\gamma$ as long as $\gamma<\hat{\gamma}$; otherwise (for $\hat{\gamma}<\gamma<1$ ), it increases with the degree of product substitutability. Note that both $\widehat{x}$ and $\hat{\gamma}$ decrease with $n$, meaning that as the number of firm increases, the range of parameters over which the winner's profit increases with $\gamma$ enlarges.

\subsection{Proof of Proposition 3}

Proposition 3 is stated formally as follows.

(i) For all $x \in[0,1]$ and all $n \geq 2$, there exists $\widetilde{\gamma}_{C} \in(0,1)$ such that $\partial P I / \partial \gamma<0$ for $\gamma<\widetilde{\gamma}_{C}, \partial P I / \partial \gamma=0$ for $\gamma=\widetilde{\gamma}_{C}$, and $\partial P I / \partial \gamma>0$ for $\gamma>\widetilde{\gamma}_{C}$. (ii) The highest profit incentive is reached under independent products $(\gamma=0)$ if $x<\bar{x}_{C}(n) \equiv 2(n-1) /(3 n+1)$ and under homogeneous products $(\gamma=1)$ if $x>\bar{x}_{C}(n) ; \bar{x}_{C}(n)$ increases in $n$, is comprised beween $2 / 7$ and $2 / 3$.

(Proof) (i) Computing the first-order derivative of the profit incentive with respect to $\gamma$ yields

$$
\frac{\partial}{\partial \gamma} P I_{C}(n, \gamma)=\frac{2 x(n-1)}{(2-\gamma)^{3}(2+\gamma(n-1))^{3}}(A(n, \gamma) x+B(n, \gamma)),
$$


where

$$
\begin{aligned}
A(n, \gamma) & \equiv \gamma(4+(n-2) \gamma)(2+(n-2) \gamma) \\
B(n, \gamma) & \equiv 2(2-\gamma)\left((n-2) \gamma^{2}-(n-5) \gamma-2\right)
\end{aligned}
$$

The derivative has the same sign as $A(n, \gamma) x+B(n, \gamma)$. It is readily observed that $A(n, \gamma)$ is positive and increasing in $\gamma$ for all $n$ and $\gamma$. We also note that $A(n, 0)=0$ and $A(n, 1)=n(n+2)>0$.

The sign of $B(n, \gamma)$ changes. $B(n, \gamma)$ is a polynomial of degree 3 in $\gamma$; yet, as $(2-\gamma)>0$ can be factored out, we need to analyse the sign of the second-degree polynomial $\beta(n, \gamma) \equiv(n-2) \gamma^{2}-(n-5) \gamma-2$. It is easily checked that $\beta(n, \gamma)$ admits two real roots, $\gamma_{1}$ and $\gamma_{2}$, which can be ranked as follows: $\gamma_{1}<0<\gamma_{2}<1$. As $B(n, 0)=-8$ and $B(n, 1)=2$, we conclude that $B(n, \gamma)$ has only one root in the interval $[0,1]$ and that $B(n, \gamma)>0$ iff $\gamma>\gamma_{2}$.

Collecting the previous results, we have that $A(n, \gamma) x+B(n, \gamma)$ (and, hence, the derivative $\partial P I / \partial \gamma)$ is negative in $\gamma=0$, positive in $\gamma=1$, and we can state there is only one root between zero and one, say $\widetilde{\gamma}\left(>\gamma_{2}\right)$ such that $\frac{\partial}{\partial \gamma} P I_{C}(n, \gamma)>0 \Leftrightarrow \gamma>\widetilde{\gamma}$.

(ii) We compute

$$
\begin{aligned}
P I_{C}(n, 0)-P I_{C}(n, 1) & =\frac{x(x+2)}{4}-\frac{n x(2+x n)}{(n+1)^{2}}>0 \Longleftrightarrow \\
-2 n+3 x n+2+x & <0 \Longleftrightarrow x<\bar{x}_{C}(n) \equiv \frac{2(n-1)}{(3 n+1)} .
\end{aligned}
$$

We check that $\bar{x}_{C}^{\prime}(n)>0, \bar{x}_{C}^{\prime \prime}(n)<0$ and $\bar{x}_{C}(2)=2 / 7$ and $\lim _{n \rightarrow \infty} \bar{x}_{C}(n)=$ $2 / 3$.

\subsection{Proof of Proposition 4}

Proposition 4 is formally stated as follows.

Define

$$
\begin{aligned}
& \hat{n}(\gamma)=\frac{2 \gamma(1-\gamma) x+(3 \gamma-2)(2-\gamma)}{\gamma(2-\gamma-x \gamma)}, \\
& \hat{x}(\gamma)=\frac{2(2-\gamma)\left(-\gamma^{2}-2 \gamma+4\right)}{\gamma\left(8-\gamma^{2}\right)}
\end{aligned}
$$


(i) Taking $n$ as a continuous variable, the profit incentive reaches a maximum for $n=\hat{n}(\gamma) .{ }^{11}$ (ii) Taking into account that $n$ is an integer, the maximum is reached for $n>1$ if and only if $x>\hat{x}(\gamma)$, which supposes that $\gamma>0.711$

(Proof) (i) We compute

$$
\begin{aligned}
\frac{\partial}{\partial n} P I_{C}(n, \gamma) & =2 \gamma x \frac{-4+8 \gamma+2 \gamma x-3 \gamma^{2}-2 \gamma^{2} x-2 \gamma n+\gamma^{2} n+x \gamma^{2} n}{(-2+\gamma)^{2}(2+\gamma(n-1))^{3}} \geq 0 \Leftrightarrow \\
n & <\frac{2 \gamma(1-\gamma) x+(3 \gamma-2)(2-\gamma)}{\gamma(2-\gamma-x \gamma)} \equiv \hat{n}(\gamma) .
\end{aligned}
$$

(ii) This is clearly impossible if $\hat{n}(\gamma) \leq 1$, which is equivalent to $x \leq$ $2(1-\gamma) / \gamma$. As $x<1$, the latter condition is always satisfied if $\gamma \leq 2 / 3$. Therefore, to have $P I_{C}(n, \gamma)$ reaching a maximum at a value of $n$ strictly larger than one, we need $\gamma>2 / 3$ and $x>2(1-\gamma) / \gamma$. However, recalling that $n$ is an integer, it might the case that $1<\hat{n}(\gamma)<2$ while $P I_{C}(1, \gamma)<$ $P I_{C}(2, \gamma)$. To exclude this case, we note that $P I_{C}(2, \gamma)>P I_{C}(1, \gamma) \Longleftrightarrow$

$$
x>\frac{2(2-\gamma)\left(-\gamma^{2}-2 \gamma+4\right)}{\gamma\left(8-\gamma^{2}\right)} \equiv \hat{x}(\gamma),
$$

which supposes that $\gamma>0.711$. In sum, the maximum value of PI is reached for $n=1$ (monopoly) if $x \leq \hat{x}(\gamma)$ (which is always true for $\gamma \leq 0.711$ ).

\subsection{Proof of Proposition 5}

Let us first prove Expression (10):

$$
\frac{\partial}{\partial n} P I_{C}(n, \gamma)=2 \gamma x \frac{(n+x n-3-2 x) \gamma^{2}+(8+2 x-2 n) \gamma-4}{(2-\gamma)^{2}(2+\gamma(n-1))^{3}}>0
$$

if and only if, for $\gamma>0,(n+x n-3-2 x) \gamma^{2}+(8+2 x-2 n) \gamma-4>0$. This quadratic form in $\gamma$ has one negative and one positive root, $\bar{\gamma}(n, x)$. Therefore, $\partial P I_{C}(n, \gamma) / \partial n>0$ if and only if

$$
\gamma>\bar{\gamma}(n, x)=\frac{n-x-4+\sqrt{(n+x)^{2}-4(n-1)}}{n-3+n x-2 x},
$$

which supposes that $x>(n-1) / n$ (otherwise, $\bar{\gamma}(n, x)>1$ ).

\footnotetext{
${ }^{11}$ Computing $\hat{n}(\gamma)$ at $\gamma=1$, we find that the profit incentive decreases with $n$ as long as $n>1 /(1-x)$; this is equivalent to $x<(n-1) / n$, the condition given by Yi (1999).
} 
Second, we compute a lower bound on $\bar{\gamma}(n, x)$. As $\bar{\gamma}(n, x)$ decreases with $x$, we evaluate $\bar{\gamma}(n, x)$ at $x=1$ and we obtain

$$
\bar{\gamma}(n, 1)=\frac{n-5+\sqrt{n^{2}-2 n+5}}{2 n-5},
$$

which is an increasing function of $n$. The lowest value is therefore reached for $n=2$; that is $\bar{\gamma}(2,1)=3-\sqrt{5}=0.764$, which is our lower bound.

Third, we evaluate $\partial P I_{C}(n, \gamma) / \partial \gamma$ at the lower bound we have just found. We have

$$
\left.\frac{\partial}{\partial \gamma} P I_{C}(n, \gamma)\right|_{\gamma=\frac{764}{1000}}>0 \Longleftrightarrow x>\frac{618}{191} \frac{11269 n-40788}{(618+191 n)(118+191 n)} .
$$

But, by assumption, $x>(n-1) / n$ and

$$
\begin{aligned}
\frac{n-1}{n}-\frac{618}{191} \frac{11269 n-40788}{(618+191 n)(118+191 n)} & >0 \Longleftrightarrow \\
12285452 n+12917903 n^{2}+6967871 n^{3}-13928484 & >0 .
\end{aligned}
$$

The latter expression increases in $n$. Evaluated at $n=2$, it is equal to 118057000 , meaning that the expression is positive for all $n \geq 2$.

We conclude that $\partial P I_{C}(n, \gamma) / \partial \gamma$ is positive at $\bar{\gamma}(n, x)$ for all $n$ and $x$, which implies that $\bar{\gamma}(n, x)>\tilde{\gamma}(n, x)$ (as $P I_{C}(n, \gamma)$ is U-shaped in $\gamma$ ) and completes the proof.

\subsection{Proof of Lemma 6}

The post-innovation price game is played by one winner (indexed by $W$ ) and a set $L$ of $(n-1)$ losers indexed by $i$. A typical loser $i$ 's maximization program writes as

$$
\max _{p_{i}}\left(\alpha-\beta p_{i}+\delta p_{W}+\delta \sum_{j \in L, j \neq i} p_{j}\right)\left(p_{i}-c\right) \text { s.t. } p_{i} \geq c .
$$

From the FOC, we find that the interior solution to this problem is given by

$$
p_{i}=\frac{1}{2 \beta}\left(\alpha+\delta p_{W}+\delta \sum_{j \in L, j \neq i} p_{j}+\beta c\right) .
$$


This solution holds as long as $p_{i} \geq c$, which is equivalent to

$$
\alpha+\delta p_{W}+\delta \sum_{j \in L, j \neq i} p_{j} \geq \beta c .
$$

We can thus write loser $i$ 's best response function as follows

$R_{i}\left(p_{W},\left(p_{j}\right)_{j \in L}^{j \neq i}\right)= \begin{cases}\frac{1}{2 \beta}\left(\alpha+\delta p_{W}+\delta \sum_{j \in L, j \neq i} p_{j}+\beta c\right) & \text { if }(14) \text { is met } \\ c & \text { otherwise. }\end{cases}$

As for the winner, the maximization program writes as

$$
\max _{p_{W}}\left(\alpha-\beta p_{W}+\delta \sum_{i \in L} p_{i}\right)\left(p_{W}-c+x\right) \text { s.t. } p_{W} \geq c-x .
$$

From the FOC, we find that the interior solution to this problem is given by

$$
p_{W}\left(\left(p_{i}\right)_{i \in L}\right)=\frac{1}{2 \beta}\left(\alpha+\delta \sum_{i \in L} p_{i}+\beta(c-x)\right) .
$$

We show that this value is always larger than $c-x$. Indeed, the lowest of $p_{w}$, noted $\underline{p_{w}}$, is reached when $p_{i}=c \forall i \in L$. After substitutions, we compute

$$
\underline{p_{W}}-(c-x)=\frac{1}{2} \frac{(1-\gamma)+x(1+\gamma n-2 \gamma)}{1+\gamma n-2 \gamma}>0 .
$$

Let us first characterize the interior Nash equilibrium. Solving the system of equations given by (16) and by the top row of (15) taken $(n-1)$ times, we get equilibrium prices for the winner and the losers respectively given by $p_{W}^{B}$ and $p_{L}^{B}$. We have that

$$
\begin{aligned}
p_{L}^{B}-c & =\frac{(1-\gamma)(2 \gamma n-3 \gamma+2)-\gamma(1+\gamma n-2 \gamma) x}{(2+\gamma n-3 \gamma)(2+2 \gamma n-3 \gamma)} \geq 0 \\
& \Longleftrightarrow x \leq x_{B}(n, \gamma)=\frac{(1-\gamma)(2 \gamma n-3 \gamma+2)}{\gamma(1+\gamma n-2 \gamma)} .
\end{aligned}
$$

Suppose now that condition (17) is not met. The equilibrium must then be such that some losers set $p_{i}=c$, while the other losers set a price $\hat{p}_{L}>c$. Suppose that $k$ losers price above marginal cost, with $0<k<(n-1)$. Condition (14) must be satisfied for those $k$ losers and violated for the other $(n-k-1)$ ones. That is,

$$
\begin{aligned}
\alpha+\delta p_{W}+\delta\left((n-k-1) c+(k-1) \hat{p}_{L}\right) & \geq \beta c, \\
\alpha+\delta p_{W}+\delta\left((n-k-2) c+k \hat{p}_{L}\right) & <\beta c .
\end{aligned}
$$


As $\hat{p}_{L}>c$, it is easily seen that these two inequalities cannot be met simultaneously. We therefore conclude that the only corner equilibrium involves $k=n-1$.

Now, the winner prices its good at the maximum level leaving the losers with zero demand at their lowest possible price, i.e., $p_{i}=c \forall i \in L$. That is, $\bar{p}_{W}^{B}$ is such that

$$
q_{L}^{B}=\alpha-(\beta-\delta(n-1)) c+\delta p_{W}=0 .
$$

After substituting for the values of $\alpha, \beta$, and $\delta$, one gets

$$
\begin{aligned}
& \bar{p}_{W}^{B}=a-\frac{1}{\gamma}(a-c), \\
& \bar{q}_{W}^{B}=a-\bar{p}_{W}^{B}=\frac{1}{\gamma}(a-c)>0
\end{aligned}
$$

Accordingly, the winner's profit is computed as (recalling that we set $a-c \equiv$ $1)$ :

$$
\bar{\pi}_{W}^{B}(\gamma)=\left(1-\frac{1}{\gamma}+x\right) \frac{1}{\gamma}
$$

One checks that when $\gamma$ tends to $1, \bar{p}_{W}^{B}$ tends to $c$ (the loser's marginal cost) and $\bar{\pi}_{W}^{B}$ tends to $x$. Note also that in the corner solution, the winner's profit becomes independent of $n$.

\subsection{Proof of Proposition 7}

(i) Consider first the case where all firms stay on the market after the innovation. This is so as long as $x \leq x_{B}(n, \gamma)$, which is equivalent to $\gamma \leq \gamma_{B}(n)$, with

$$
\gamma_{B}(n)=\frac{1}{2(x n-2 x+2 n-3)}\left(2 n-x-5+\sqrt{x(x+4 n-6)+(2 n-1)^{2}}\right)
$$

Notice that $\gamma_{B}(n)$ is decreasing in $n$, and so $\min \gamma_{B}(n)=2 /(x+2)$. The profit incentive is then computed as follows: $P I_{B}(n, \gamma)=\pi_{W}(n, \gamma)-\pi(n, \gamma)$ and, recalling from $(3)$ that $\pi_{i}^{B}=(1 / \beta)\left(q_{i}^{B}\right)^{2}$,

$$
\frac{\partial}{\partial \gamma} P I_{B}(n, \gamma)=\frac{\partial}{\partial \gamma}\left(\frac{1}{\beta}\left(q_{W}+q\right)\right)\left(q_{W}-q\right)+\frac{\partial}{\partial \gamma}\left(q_{W}-q\right)\left(\frac{1}{\beta}\left(q_{W}+q\right)\right) .
$$


Developping, we have that:

$$
\begin{aligned}
\frac{\partial}{\partial \gamma}\left(\frac{1}{\beta}\left(q_{W}+q\right)\right) & =-(n-1) \frac{\gamma(3 \gamma(n-2)+4) x+2(2+\gamma(2 n-3))^{2}}{(2+2 \gamma n-3 \gamma)^{2}(2+\gamma n-3 \gamma)^{2}}<0, \\
\frac{\partial}{\partial \gamma}\left(q_{W}-q\right) & =x \cdot \frac{\partial}{\partial \gamma} G(\gamma, n)>0, \text { with } \\
G(\gamma, n) & =\frac{(1+\gamma n-2 \gamma)\left(2+\gamma^{2} n^{2}-6 \gamma+5 \gamma^{2}-5 \gamma^{2} n+3 \gamma n\right)}{(2+\gamma n-3 \gamma)(2+2 \gamma n-3 \gamma)(1-\gamma)(1+\gamma n-\gamma)}
\end{aligned}
$$

Therefore, as $\gamma$ increases two opposite forces are at work. Computing $\partial P I_{B}(n, \gamma) / \partial \gamma$ at the extreme values $\left(\gamma=0\right.$ and $\left.\gamma=\min \gamma_{B}\right)$, we find that $\partial P I_{B}(n, 0) / \partial \gamma<0$ and $\partial P I_{B}(n, 2 /(2+x)) / \partial \gamma \geq 0$. Given the opposite forces involved and given that the profit incentive is first decreasing and then increasing in $\gamma$, we can conclude that the PI has a U-shape w.r.t. $\gamma \cdot{ }^{12}$

(ii) Second, for $x>x_{B}(n, \gamma)$, which is equivalent to $\gamma>\gamma_{B}(n)$, the profit incentive is defined as $\overline{P I}_{B}(n, \gamma)=\bar{\pi}_{W}^{B}(\gamma)-\pi(n, \gamma)$. As it can be easily shown that $\bar{\pi}_{W}^{B}(\gamma)$ increases with $\gamma$ and $\pi(n, \gamma)$ decreases with $\gamma$, we can safely conclude that $\overline{P I}_{B}(n, \gamma)$ is an increasing function of $\gamma$.

Now, we can compute the limit of $\overline{P I}_{B}(n, \gamma)$ for $\gamma \rightarrow 1$ :

$$
\lim _{\gamma \rightarrow 1} \overline{P I}_{B}(n, \gamma)=x
$$

Comparing the latter expression to $P I_{B}(n, 0)$, we have that $\overline{P I}_{B}(n, \gamma)-$ $P I_{B}(n, 0)=x(2-x) / 4>0$. (by the way, $\overline{P I}_{B}(n, \gamma)$ as $\gamma$ approaches one is the profit incentive for a perfectly competitive firm and is, as we know from Arrow, larger than the profit incentive for a monopolist, i.e., $\left.P I_{B}(n, 0)=P I_{B}(1, \gamma)\right)$.

\subsection{Proof of Proposition 8}

We first note that the profit incentive is independent of $n$ for $\gamma=0$ (as firms are local monopolists). For $\gamma>0$, we distinguish between two cases.

(i) Consider first the case where $x \leq x_{B}(n, \gamma)$ and all firms stay on the market after the innovation. The profit incentive is given by $P I_{B}(n, \gamma)=$

\footnotetext{
${ }^{12}$ If $\partial P I_{B} / \partial \gamma$ is negative in $\gamma=0$ and positive in $\min \gamma_{B}$, given the two opposite forces, it means that in $\min \gamma_{B}$ the derivative has already changed its sign and this is sufficient to prove that it is increasing also for any $\gamma>\min \gamma_{B}$.
} 
$\pi_{W}^{B}(n, \gamma)-\pi^{B}(n, \gamma)$. Computing the derivative with respect to $n$ and solving for $x$, we obtain: ${ }^{13}$

$$
\frac{\partial P I_{B}(n, \gamma)}{\partial n}>0 \Longleftrightarrow x>\bar{x}(n, \gamma)
$$

We now show that $\bar{x}(n, \gamma)<x_{B}(n, \gamma)$. Computing the difference $\bar{x}(n, \gamma)-$ $x_{B}(n, \gamma)$, we find

$$
\bar{x}(n, \gamma)-x_{B}(n, \gamma)=-\frac{(1-\gamma)(2 \gamma n-3 \gamma+2)\left(A_{5} \gamma^{5}+A_{4} \gamma^{4}+A_{3} \gamma^{3}+A_{2} \gamma^{2}+A_{1} \gamma+4\right)}{B},
$$

where

$$
\begin{aligned}
A_{5} & =3 n^{5}-27 n^{4}+101 n^{3}-195 n^{2}+191 n-75 \geq 0 \Longleftrightarrow n \geq 3, \\
A_{4} & =-106 n^{3}+206+312 n^{2}-414 n+14 n^{4}>0, \\
A_{3} & =-163 n^{2}-224+332 n+27 n^{3}>0 \Longleftrightarrow n \geq 2, \\
A_{2} & =122+28 n^{2}-118 n>0 \text { for all } n \neq 2, \\
A_{1} & =16 n-34>0 \Longleftrightarrow n \geq 3, \\
B & >0 .
\end{aligned}
$$

We thus have that $\bar{x}(n, \gamma)-x_{B}(n, \gamma)<0$ for $n \geq 3$. To complete the proof, we compute the value of $A_{5}(n) \gamma^{5}+A_{4}(n) \gamma^{4}+A_{3}(n) \gamma^{3}+A_{2}(n) \gamma^{2}+$ $A_{1}(n) \gamma+4$ at $n=1$ and $n=2$ and we find, respectively, $2(2-\gamma)(\gamma-1)^{4}>$ 0 and $4-\gamma^{5}+2 \gamma^{4}+4 \gamma^{3}-2 \gamma^{2}-2 \gamma>0$.

So, for $0<x<\bar{x}(n, \gamma), \partial P I_{B}(n, \gamma) / \partial n<0$, and for $\bar{x}(n, \gamma) \leq x \leq$ $x_{B}(n, \gamma), \partial P I_{B}(n, \gamma) / \partial n \geq 0$. Simulations show that a necessary condition for $\bar{x}(n, \gamma)<1$ is $\gamma>0.65$.

(ii) If $x>x_{B}(n, \gamma)$, the profit incentive is defined as $\overline{P I}_{B}(n, \gamma)=$ $\bar{\pi}_{W}^{B}(\gamma)-\pi(n, \gamma)$. In this case, it is easily shown that $\overline{P I}_{B}(n, \gamma)$ is increasing in $n$, because $\bar{\pi}_{W}^{B}(\gamma)$ is independent of $n$ and $\pi(n, \gamma)$ decreases with $n$. At the extreme case of $\gamma=1, \overline{P I}_{B}(n, 1)=x$, which is independent of $n$.

\footnotetext{
${ }^{13}$ The long expressions are not reproduced here but are available from the authors upon request.
} 


\subsection{Proof of Proposition 9}

Under Cournot competition, we know from Proposition 4 that the optimal number of firms $n_{C}^{*}$ and the corresponding profit incentives are

$$
\left\{\begin{array}{lll}
\text { for } x \leq \hat{x}(\gamma), & n_{C}^{*}=1, & P I_{C}(1, \gamma)=\frac{1}{4}(x+2) x, \\
\text { for } x>\hat{x}(\gamma), & n_{C}^{*}=\hat{n}(\gamma), & P I_{C}(\hat{n}(x, \gamma), \gamma)=\frac{x}{(4-x \gamma-2 \gamma) \gamma}
\end{array}\right.
$$

with

$$
\begin{aligned}
\hat{n}(\gamma) & =\frac{2 \gamma(1-\gamma) x+(3 \gamma-2)(2-\gamma)}{\gamma(2-\gamma-x \gamma)}, \\
\hat{x}(\gamma) & =\frac{2(2-\gamma)\left(-\gamma^{2}-2 \gamma+4\right)}{\gamma\left(8-\gamma^{2}\right)} .
\end{aligned}
$$

Under Bertrand competition, we know from Proposition 8 that the profit incentive decreases with the number of firms for $x<\bar{x}(n, \gamma)$, and increases otherwise. So, for $x<\bar{x}(n, \gamma), n_{B}^{*}=1$ and $P I_{B}(1, \gamma)=(x+2) x / 4$. On the other hand, for $x>\bar{x}(n, \gamma), n_{B}^{*}=\infty$. The level of the profit incentive depends then on whether $x$ is below or above $x_{B}(n, \gamma)$. Computing $\bar{x}(n, \gamma)$ and $x_{B}(n, \gamma)$ for $n \rightarrow \infty$, we find that they both tend to the same value, i.e., $2(1-\gamma) / \gamma$. Summarizing, we have

$$
\left\{\begin{array}{lll}
\text { for } x \leq \frac{2(1-\gamma)}{\gamma}, & n_{B}^{*}=1, & P I_{B}(1, \gamma)=\frac{1}{4}(x+2) x \\
\text { for } x>\frac{2(1-\gamma)}{\gamma}, & n_{B}^{*}=\infty, & \overline{P I}_{B}(\infty, \gamma)=\left(1-\frac{1}{\gamma}+x\right) \frac{1}{\gamma} .
\end{array}\right.
$$

We establish that

$$
\hat{x}(\gamma)-\frac{2(1-\gamma)}{\gamma}=\frac{2 \gamma}{8-\gamma^{2}}>0,
$$

which implies that there are three cases to consider:

- for $x<\frac{2(1-\gamma)}{\gamma}, n_{C}^{*}=n_{B}^{*}=1$ and monopoly should prevail;

- for $\frac{2(1-\gamma)}{\gamma}<x<\hat{x}(\gamma)$, Bertrand competition should prevail because

$$
P I_{B}(\infty, \gamma)-P I_{C}(1, \gamma)=\frac{1}{4}\left(x-\frac{2(1-\gamma)}{\gamma}\right) \frac{2-x \gamma}{\gamma^{3}}>0
$$

- for $x>\hat{x}(\gamma)$, Bertrand competition should prevail because

$$
P I_{B}(\infty, \gamma)-P I_{C}(\hat{n}(x, \gamma), \gamma)=\left(x-\frac{2(1-\gamma)}{\gamma}\right) \frac{2-x \gamma-\gamma}{\gamma^{3}(4-x \gamma-2 \gamma)}>0
$$




\section{References}

[1] Amir, R., Evstigneev, I. and Wooders, J. (2003). Noncooperative versus cooperative R\&D with endogenous spillover rate. Games and Economic Behavior 42:183-207.

[2] Arrow, K. (1962). Economic welfare and the allocation of resources for inventions, in The rate and direction of inventive activity. R. Nelson (ed.). Princeton University press.

[3] Bester, H. and Petrakis, E. (1993). The incentives for cost reduction in a differentiated industry, International Journal of Industrial Organization 11: $519-34$.

[4] Bonanno, G. and Haworth, B. (1998). Intensity of competition and the choice between product and process innovation. International Journal of Industrial Organization 16: 495-510.

[5] Boone, J. (2001). Intensity of competition and the incentive to innovate. International Journal of Industrial Organization 19: 705-726.

[6] Brander, J. and Spencer, B. (1983). Strategic commitment with R\&D: the symmetric case. Bell Journal of Economics 14, 225-235.

[7] d'Aspremont, C. and Jacquemin, A. (1988). Cooperative and noncooperative R\&D in duopoly with spillovers. American Economic Review 78: 1133-1137. Erratum: American Economic Review 80 (1990): 641642.

[8] Delbono, F. and Denicolo, V. (1990). R\&D investment in a symmetric and homogeneous oligopoly. International Journal of Industrial Organization 8: 297-313.

[9] Kamien, M.I. (1992). Patent licensing, in Handbook of Game Theory. R.J. Aumann and S. Hart (eds.) Vol. 1: 332-354.

[10] Kamien, M.I., Muller, E., and Zang, I. (1992). Reserach joint ventures and R\&D cartels. American Economic Review 82: 1293-1306. 
[11] Kamien, M.I. and Tauman, Y. (2002). Patent licensing: The inside story. The Manchester School 70: 7-15.

[12] Lin, P. and Saggi, K. (2002). Product differentiation, process R\&D, and the nature of market competition. European Economic Review 46: 201-211.

[13] Okuno-Fujiwara, M. and Suzumura, K. (1990). Strategic cost-reduction investment and economic welfare. Mimeo. IER, Hitotsubashi Univ.

[14] Qiu, L. D. (1997). On the dynamic efficiency of Bertrand and Cournot equilibria, Journal of Economic Theory 75: 213 - 29.

[15] Schumpeter, J. (1943). Capiatlism, socialism, and democracy. Unwin University Books, London.

[16] Scotchmer, S. (2004). Innovation and incentives. MIT Press, Cambridge.

[17] Singh, N. and Vives, X. (1984). Price and quantity competition in a differentiated duopoly. RAND Journal of Economics 15: 546-554.

[18] Spence, M. (1984). Cost reduction, competition and industry performance. Econometrica 52, 101-112.

[19] Yi, S.-S. (1999). Market structure and the incentives to innovate: the case of Cournot oligopoly. Economics letters 65: 379-388.

[20] Zanchettin, P. (2004). Differentiated duopoly with asymmetric costs: new results from a seminal paper. Mimeo. University of Nottingham. 
Département des Sciences Économiques de l'Université catholique de Louvain

Institut de Recherches Économiques et Sociales

Place Montesquieu, 3

1348 Louvain-la-Neuve, Belgique 